Technical Reports

\title{
Corporate Network Analysis with IPTV Traffic Insertion
}

\author{
${ }^{1}$ Filipe Hiluy Lima, ${ }^{2}$ Henrique Mariano Costa do Amaral and ${ }^{3}$ Denílson Moreira Santos \\ ${ }^{1,2}$ Department of Computer Engineering, State University of Maranhão, São Luis, Brasil \\ ${ }^{3}$ Department of Design and Technology, Federal University of Maranhão, São Luis, Brasil
}

Article history

Received: 29-3-2015

Revised: $22-4-2015$

Accepted: 24-6-2015

Corresponding Author:

Filipe Hiluy Lima

Department of Computer

Engineering, State University

of Maranhão,

São Luis, Brasil

Email: fhiluy@hotmail.com

\begin{abstract}
This paper presents studies about Internet Protocol Television (IPTV) technology starting with its motivation that led to its birth and its great and current application. The article theoretical basis will present the description of mechanisms, standards and main protocols used to ensure a quality of service needed to the deployment of IPTV applications. Among them, we will mention Multiprotocol Label Switching (MPLS). It will be made a case study, including a corporate network of a large a mining company, which simulates the core of a provider network between data concentrator branches. The aim of this simulation is to analyze the network behavior after ITPV traffic insertion and serve as a basis to obtain conclusions about the possibility of developing new applications that use this technology.
\end{abstract}

Keywords: IPTV, QoS, MPLS

\section{Introduction}

With the ever increasing necessity of multimedia files transference, there is a natural need of evolution in providing such services, what can be perceived in opening a range of options, among them the appearance of television broadcasting via digital way, that is, television signals digitalization and transference through computers Internet Protocol (IP) network. An advantage in researching Internet Protocol TeleVision (IPTV) platform is the predominant basis on software with the transport of its contents through the set of IP internet protocols that ensures an implementation of service quality (Shihab and Cai, 2007; Tanenbaum, 2003; Uzunalioglu, 2009; Vieira and Maurício, 2010).

The IPTV platform can be offered or implemented for any user who with an adequate infrastructure of robust network with minimum speed for transmission. Therefore, in corporate area, this service has being adopted and aligned for automation services and monitoring remote areas, telepresence meetings and etc.

In IPTV platform infrastructure, it includes encoders, decoders, middleware equipment, videos and the equipment that creates these videos. A width of minimum band is required to obtain a good transmission for the user. The most used media standard, today, is the Moving Pictures Expert Group (MPEG-2), which requires between 3-9 Mbps of bandwidth (Zeadally et al., 2011).

Nowadays, corporate companies are increasingly being adapted to technological advances and taking advantage of opportunities to add value to the services offered in a more innovative and effective way. This paper proposes to make a viability study on the implementation of applications that use IPTV technology. It will be made a comparison of current and future consumption of bandwidth in the core of data network of the company, what will enable a behavior analysis about this new setting.

\section{Standards, Architecture and Protocols}

To create a television system over IP, it is necessary that its architecture is separated into some layers needed for services grouping and well-defined files. Nowadays, these layers are covered by standards, reflecting in an effort to interoperate several market IPTV systems. The standards are specifically understood as coding the videos that will be sent. The delivery of multimedia services, both interactive and personalized, are objects of standardization for several institutions, such as, International Telecommunication UnionTelecommunication (ITU-T) and Open IPTV Forum (Yarali and Cherry, 2005).

The ITU-T institution has a group formed to accomplish the mission to globally standardize IPTV services. This group is based on the consumer-server architecture with the additional of services delivery platform, considering the areas of digital rights record, Quality of Service $(Q o S)$ and Quality of Experience $(Q o E)$. The quality of service is presented 
as set of activities which ensure the optimization of the logical network for delivering the IPTV packets, due to the packet loss, speed of delivery and end-toend delay. Among these activities, it is included from implementation of specific protocols as MPLS and Multicast protocols, to policies of traffic prioritization or architectures of integrated and differentiated services. Whereas the quality of experience translates all technical efforts applied in the network through the broad perception of the user of this service.

A very important institution inserted in the process of this standardization is Telecoms \& Internet Converged Services \& Protocols for Advanced Networks (TISPAN) that is a group of work of European Telecommunications Standards Institute (ETSI), which defines IPTV service as the next generation of networks, that is, Next Generation Networks (NGN). Another important contribution of this process is in the inclusion by Open IPTV Forum of legacy network architectures, integrating the totality of wired networks (Zeadally et al., 2011).

For the transmission of IPTV services, it is made important mention to Digital Video Broadcast (DVB), standard document that specifies the first intentions of specification for live video and radio transmission, possessing or not interaction its control. The most popularly accepted and open for improvements codec (encoding algorithm) is MPEG-2 Systems, which is focused on the combination of elementary stream of audio and video produced by IPTV architecture. However, MPEG works in very relevant different specifications for IPTV as MPEG-E, MPEG-7 and MPEG-21. Besides, it has a component in relation to the improvement in multimedia resources management for consumption, protection and creation (Zeadally et al., 2011).

The architecture of an IPTV platform can be included in the following layers:

- A home network: This network is in the user's side who has a gateway equipment (a device that interconnects networks) serving as a kind of modem to connect one or more Set-Top-Boxes (converters), to then decode IPTV channels for exhibition and control in accessing

- An access network: The access network serves as connectivity provider for a users' home network. In this network it is found the equipment of link layer as switches of Ethernet networks to execute improved functions of interoperabilities, multicast protocols support and etc. There are several access techniques as an option of very-high-bitrate digital subscriber line ( $S W V D S L$ )

- Regional aggregation networks: They are networks represented by large data providers, that is, a network consisting of a large mesh of packets or data communications equipment, mainly from the network layer. This equipment is mainly represented by the router capable of connecting autonomous systems of other networks facilitating the information traffic

The Fig. 1 shows the representation of a basic IPTV network architecture.

From this figure, we can clearly identify each of the basic layers that an IPTV architecture or technology platform needs to be able to offer videos services.

This paper will be occupied in studying the traffic inserted in the regional aggregation network, that is, the "core" data network. It will be made a simulation of capacity required to the provider on the part of the company under study and later insertion of IPTV traffic for capacity analysis. It will be analyzed if the router located on the edge of the studied locality will have the transmission capacity of this new data stream, ensuring the minimal requirement of service quality for proper functioning.

The Quality of Service can be achieved through several implemented techniques and protocols. The main ones are through the IntServ (Integrated Services, solution that provides QoS with the resource reservation with RSVP protocol-Resource Reservation Protocol) architectures, in which it is ensured through native messages of PATH and RESV protocols exchange throughout the circuit extension with QoS (all equipment must have resources for this kind of connection). This is made with an admission control which verifies if the network has the needed conditions for such a measure. In large groups with implemented multicast, the reservation through RSVP protocol is accomplished from the recipient where the Set-Top-Box would perform the request compatible with its needs.

Whereas in the Differentiated Services, solution with packets prioritization (DiffServ) architecture, the data stream or the packets are selected in priorities through the Differentiated Services Code Point (DSCP) field, that is, there is no network resource reservation as in the IntServ, it only prioritizes the packets that will traffic. This mechanism becomes a simpler and more scalable implementation, for it can be treated packets of different data streams. Before companies' information policy, this architecture becomes well manageable. The DiffServ solution is defined by IETF through RFC 2475 (Reis Correa and Sodré dos Reis, 2012).

The Multiprotocol Label Switching has become a technology widely used by service provider companies and large corporations which implement WAN networks (Wide Area Networks). This is possible because it implements a packet switching technique through small labels, enabling faster forwarding between the nodes, preventing huge IP address researches in tables. 


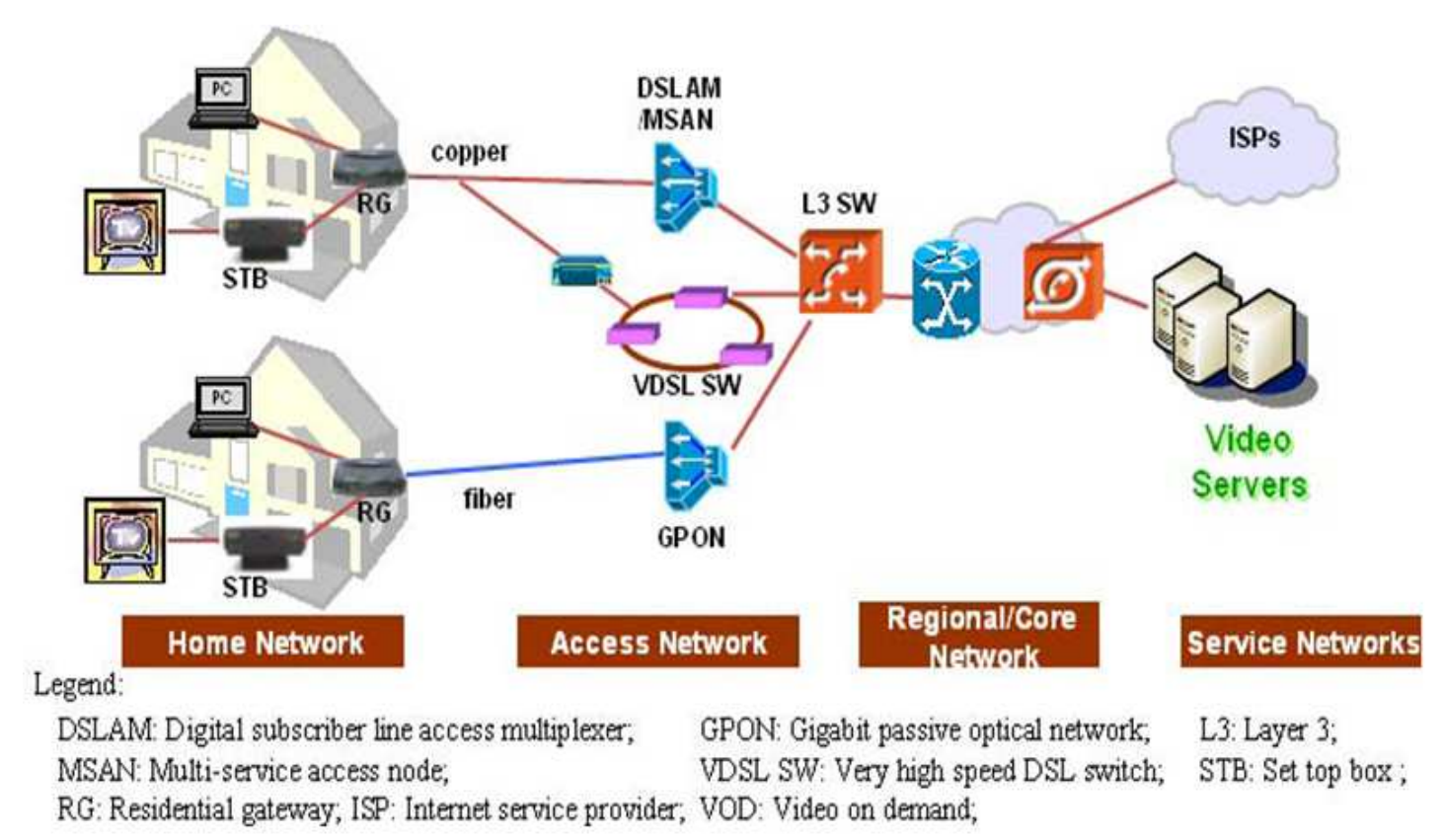

Fig. 1. Architecture of an IPTV network; Source: Wang et al. (2010)

Table 1. Services and requirements of flow, delay, jitter and packet loss

\begin{tabular}{lllll}
\hline Services/Requirements & Flow & Delay (s) & Jitter (ms) & Packet loss (\%) \\
\hline oD & 384Kbps to 1.5Mbps & 4 and 5 & 300 & $<0.1$ \\
Video (IPTV) & 1Mbps & 3 and 5 & 30 & $<0.5$ \\
Videoconference & 64Kbps to 8Mbps & 150 & 30 & $<0.5$ \\
Voice (Telephony) & 90Kbps & $150 \mathrm{~m}$ & $<=150$ & $<=0.25$ \\
\hline
\end{tabular}

Another great advantage of MPLS comes from its possibility to encapsulate packets of several network protocols, supporting a variety of technologies.

The MPLS is standardized by Internet Engineering Tasking Force (IETF) through RFC 3031. Besides serving as a mechanism for network traffic engineering, it simplifies the routers function and drastically decreases the packets overhead and latency.

The MPLS, along with the technologies and protocols present in both architectures mentioned above, acts in a complementary way, aggregating router and switching advantages, where the administrator shall choose the prioritization and technology for its configuration of better application performance.

As a consequence of quality of service application in the network, it arises a very important concept called Quality of Experience (QoE), that is, it translates the user's perception based on the experiences previously obtained in these applications. Although QoE is very subjective, there are numerical and statistical parameters about these streams over the network able to indicate the superior or inferior limit of expected quality. The most relevant QoS requirements able to show and indicate these limits are throughput (bandwidth consumption by the application), delay (maximum time of origin-destination delivery), jitter (variation in delivery delay) and packet loss (tolerance limit for application).

According to the Table 1, the requirements to ensure a good QoE in different categories of IPTV applications are the following (Barros, 2011):

The requirement flow varies due to the encoder that is used in the application, for example, the MPEG-2 needs an average of $4 \mathrm{Mbps}$ per SDTV channel. Whereas MPEG-4 uses 2Mbps per channel. The packet loss becomes very relevant in the analysis since this requirement directly compromises on transmission quality. Therefore, a percentage less than $0.5 \%$ becomes satisfactory without great damage.

\section{Methodology}

The simulation is based on the IPTV traffic insertion in a data corporate network to technically evaluate the impact this will result in its behavior and performance. This is a case study of a large corporation for insertion of a new platform of technology services, the IPTV. The implementation focus will reside in the network provider core, that is, in the aggregation networks of IPTV architecture. 


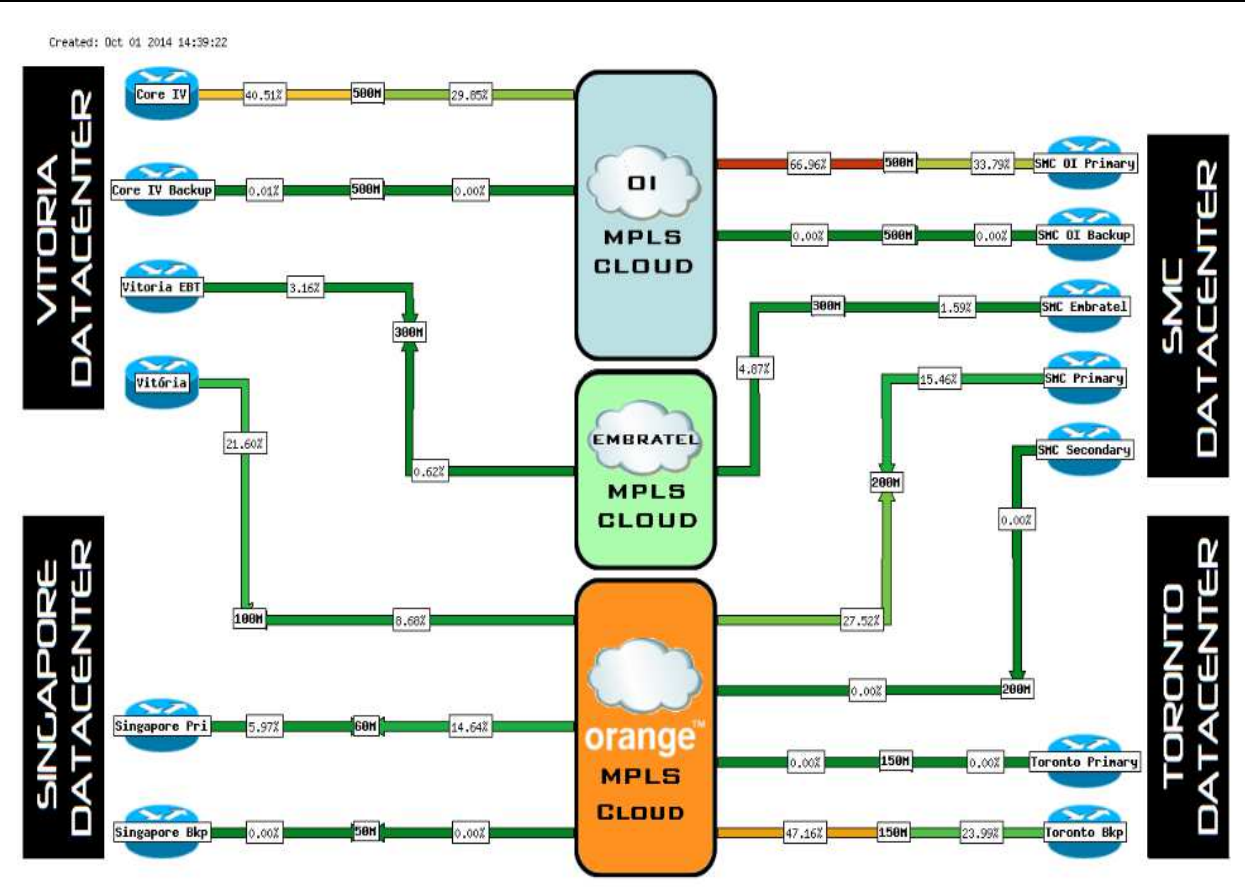

Fig. 2. Corporate topology; Source: Vale

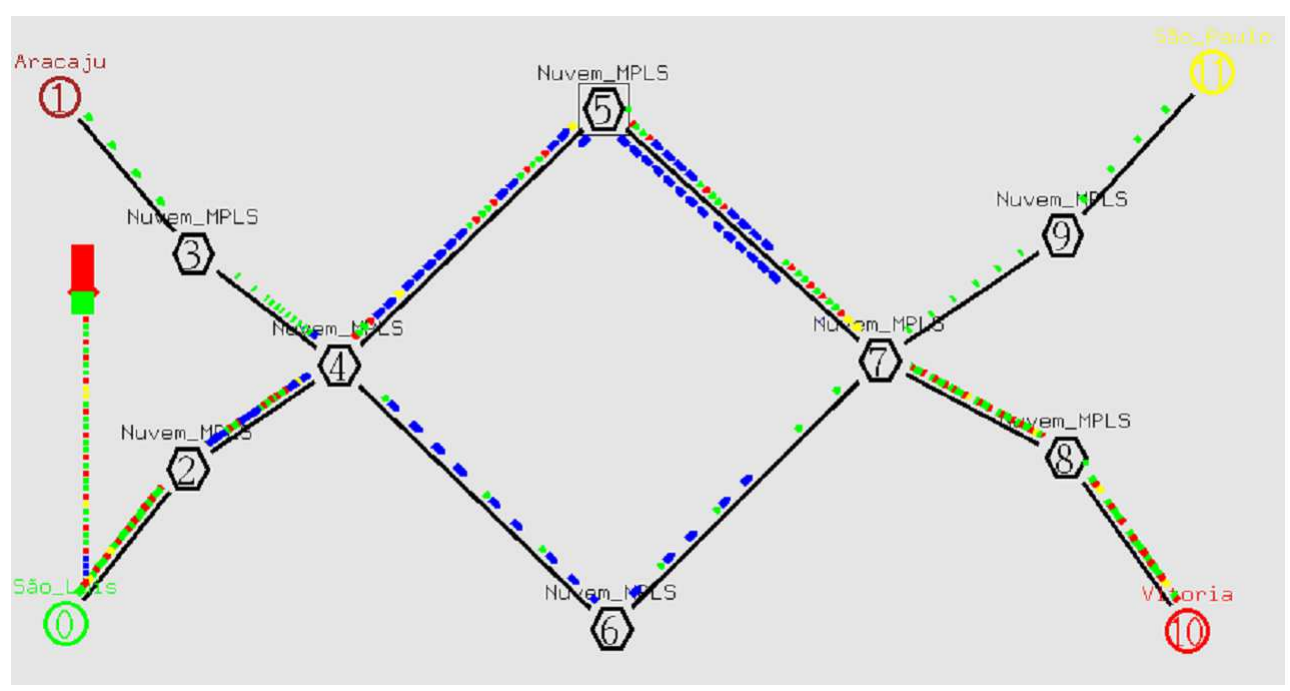

Fig. 3. Topology coded in the NS2 tool

The work methodology was achieved in distinct and successive steps. The first step consisted in requirements and parameters collection directly with the responsible for the technical IT infrastructure management (Information Technology) and for do Service Level Agreement (SLA) management with service provider. Among these requirements, it is the topology used by the company to provide the backbone (transport network) link, Fig. 2, the bandwidth used in the main links, MPLS cloud location and the traffic that is more used in the total load of the network passing bandwidth.
After this step, it was elaborated the topology taking into account all collected parameters to be made then an analysis of its current behavior. It will be based on the edge routers that connect the backbone of the towns of Vitória and São Luís. This was made through scripts (.tcl) implementations, which serve as an input in the Network Simulator 2 (NS2, simulator widely used in the academia, based on discrete events) tool, where it will compiled and generated execution logs and packets behavior representative data files over the time called trace (.tr) files. The input .tcl files will have all 
configurations related to the traffic most used by the network including the IPTV traffic.

The Fig. 3 presents the topology created in the simulation too capable to reproduce the service provider.

The MPLS cloud is represented by the routers in hexagonal format, where the labels are inserted in packets located in the router 2 input and removed at the end of the communication in the recipient (router 8 ). It is possible to verify the queue of different kinds of traffic in the router from the town of São Luís toward Vitória, passing through the way or "tunnel" of Label-Switched Path (LSP) network resources, formed by the routers 4,5 and 7 .

The log file generated by the simulator has a data matrix structure that records the network events and behavior every half a second or up to milliseconds. The simulation maximum duration time was sixty (60) seconds. This file is analyzed and processed by another application called tracegraph and NS2 Analyzer, specific for plotting the statistical graphics and results with different variables. These graphics are plotted through the insertion and reading of the entire .tr file generated, allowing filtering about the requirements to be analyzed.

One advantage of using the NS2 simulator is the utilization of discrete events, that is, the possibility to analyze the network behavior in a certain predefined period of time with insertions of variables over the interval. It becomes the closest simulation of reality due to its dynamics and power of temporal record.

Specifically, through the generation of plotted graphics and statistical data, it will be interpreted the values referring to the throughput, delay, jitter and packet loss, making an analysis of each IPTV stream inserted in the network. With these results obtained, it will be possible to say the limit of streams number that that the network has the ability to withstand. This can be concluded from the comparison made between the statistical data obtained and the values fixed in the DSL Forum Technical Report TR-126 recommendations, about the QoS requirements for IPTV services.

\section{Results}

The results were obtained from the trace files processing generated by the NS2 simulation tool. At each instance for a new IPTV traffic stream, it was made a processing for QoS requirements calculation along with its respective associated graphics.

The Table 2 shows the results grid for each instance of IPTV stream inserted in the network, associated with the corresponding QoS requirement.

All the values collected and showed in the table refer to the specific packets of IPTV applications streams that were analyzed along with other kinds of packets coming from other applications fighting for the pass band.

\section{Analyses}

Before the table presented above, we can first say that an analyzed corporate network has a tolerance and ability to process up to 03 (three) IPTV streams, ensuring the minimum requirement to obtain a good quality transmission. After the insertion of 04 (four) IPTV streams in the current network of the corporate company, there will be a natural competition with other applications so that it will happen degradation in the transmission of these IPTV packets and possibly a discrepancy in video transmission quality, in spite of being within the interval recommended for a satisfactory quality.

After this quantity inserted, it is noticeable that, from the instance of 05 (five) IPTV streams, there is a significant IPTV packets loss percentage, eliminating any investment for this number of connections.

The Fig. 4 describes an exponential curve of IPTV packets loss along the injection of new streams up to the maximum insertion of 07 (seven) streams. It can be noticed that the loss does not follow a standard or a standard numerical series among them, leaving random the percentage according to the kind of concurrent application.

The competition among the applications inserted in the corporate network will be a key point to take a measure of a more robust investment from the viewpoint of prioritizing some of them. Through the Fig. 5 and 6, it is evident that the IPTV traffic competes highly with other applications of conventional data, represented by TCP protocol streams.

The graphic presents the throughput of IPTV and TCP applications packets. This graphic makes reference to the insertion of only one IPTV stream, where it can be noticed that the competition has become evident yet for this instance and the occupation of the bandwidth follows in distinct tracks, what facilitates the nondegradation of the packets of each application.

Whereas in Fig. 6, the perception of this competition is already more evident, that is, in the limit instance for a quality of satisfactory transmission of the network, the bandwidth competition for the IPTV packets with the general data packets becomes more fierce, causes a degradation for the services and occupies the limits of transmission tracks.

With the insertion of the last instance of 07 (seven) streams of IPTV applications, there is a farther degradation of TCP packets throughput, including being exceeded in the flow of sending (Fig. 7).

With the increase in the network occupation to the detriment of the TCP packets, the applications to which the company was already operating in a satisfactory way before its clients may suffer losses of availability, instability and even data inconsistencies. On the other hand, a new IPTV technology can ensure greater productive returns from the point of view of technological possibilities increasing. 
Filipe Hiluy Lima et al. / Journal of Computer Science 2015, 11 (5): 730.737 DOI: $10.3844 /$ jessp.2015.730.737

Table 2. Simulation results

\begin{tabular}{|c|c|c|c|c|c|}
\hline Instance IPTV streams & Throughput (B/s) & Delay (s) & Jitter $(\mathrm{s})$ & Packet loss & Packet loss $(\%)$ \\
\hline 1 IPTV Stream & $71000 \mathrm{~B} / \mathrm{s}$ & 0.0847 & 0.0133 & 0 & 0.00000 \\
\hline 2 IPTV Streams & $134000 \mathrm{~B} / \mathrm{s}$ & 0.0849 & 0.0121 & 10 & 0.18113 \\
\hline 3 IPTV Streams & $179000 \mathrm{~B} / \mathrm{s}$ & 0.0862 & 0.0102 & 24 & 0.29151 \\
\hline 4 IPTV Streams & $235000 \mathrm{~B} / \mathrm{s}$ & 0.0861 & 0.0096 & 55 & 0.50510 \\
\hline 5 IPTV Streams & $287000 \mathrm{~B} / \mathrm{s}$ & 0.0868 & 0.0110 & 88 & 0.64408 \\
\hline 6 IPTV Streams & $333000 \mathrm{~B} / \mathrm{s}$ & 0.0857 & 0.0085 & 123 & 0.75082 \\
\hline 7 IPTV Streams & $374000 \mathrm{~B} / \mathrm{s}$ & 0.0881 & 0.0081 & 176 & 0.91896 \\
\hline
\end{tabular}

\section{- Packet loss}

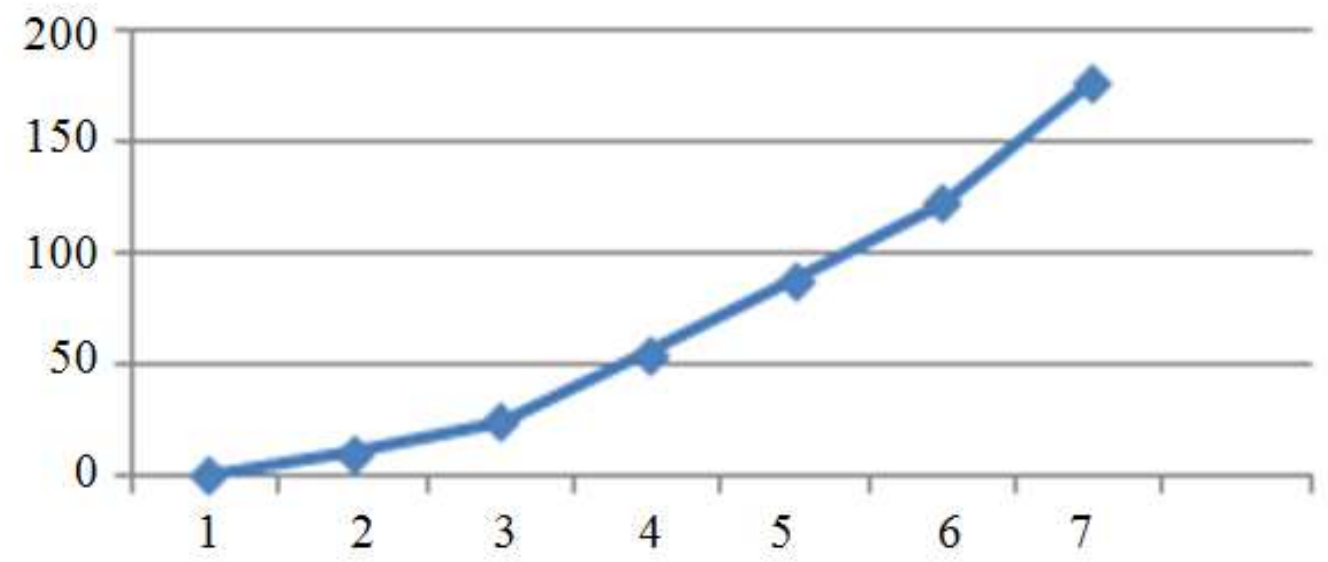

Streams

Fig. 4. Packet loss curve

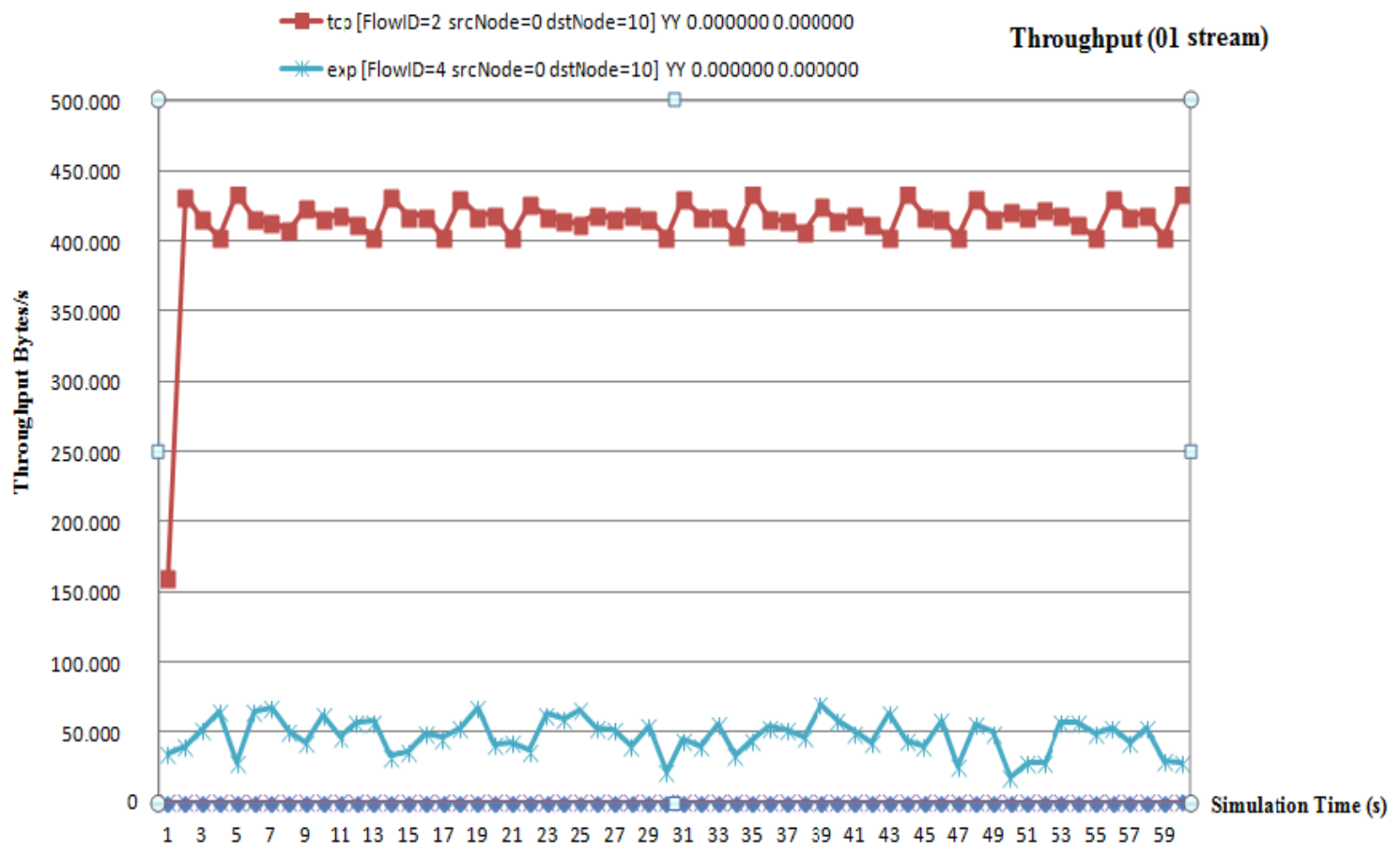

Fig. 5. Throughput IPTV x TCP (one stream) 


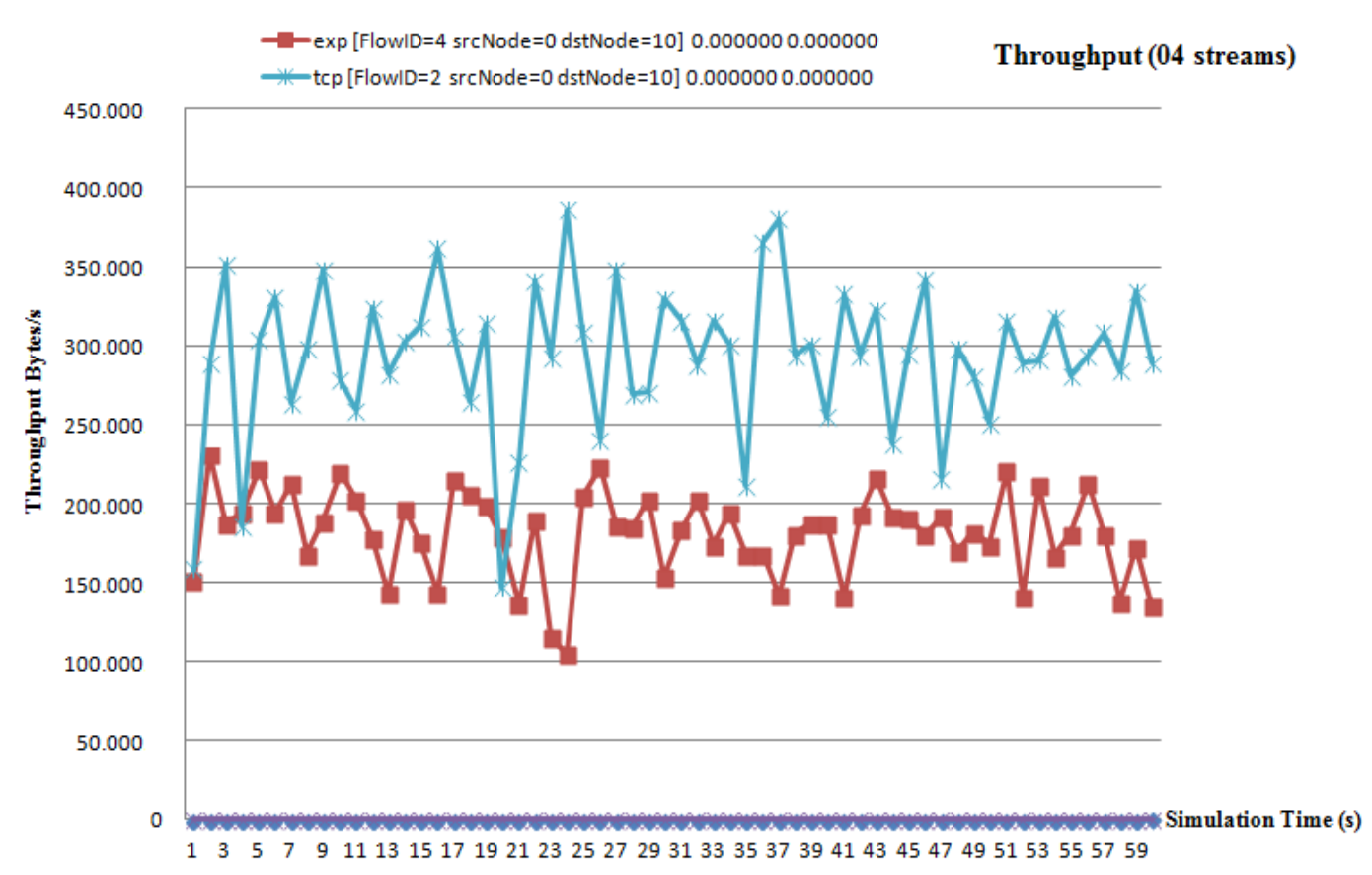

Fig. 6. Throughput IPTV x TCP (4 streams)

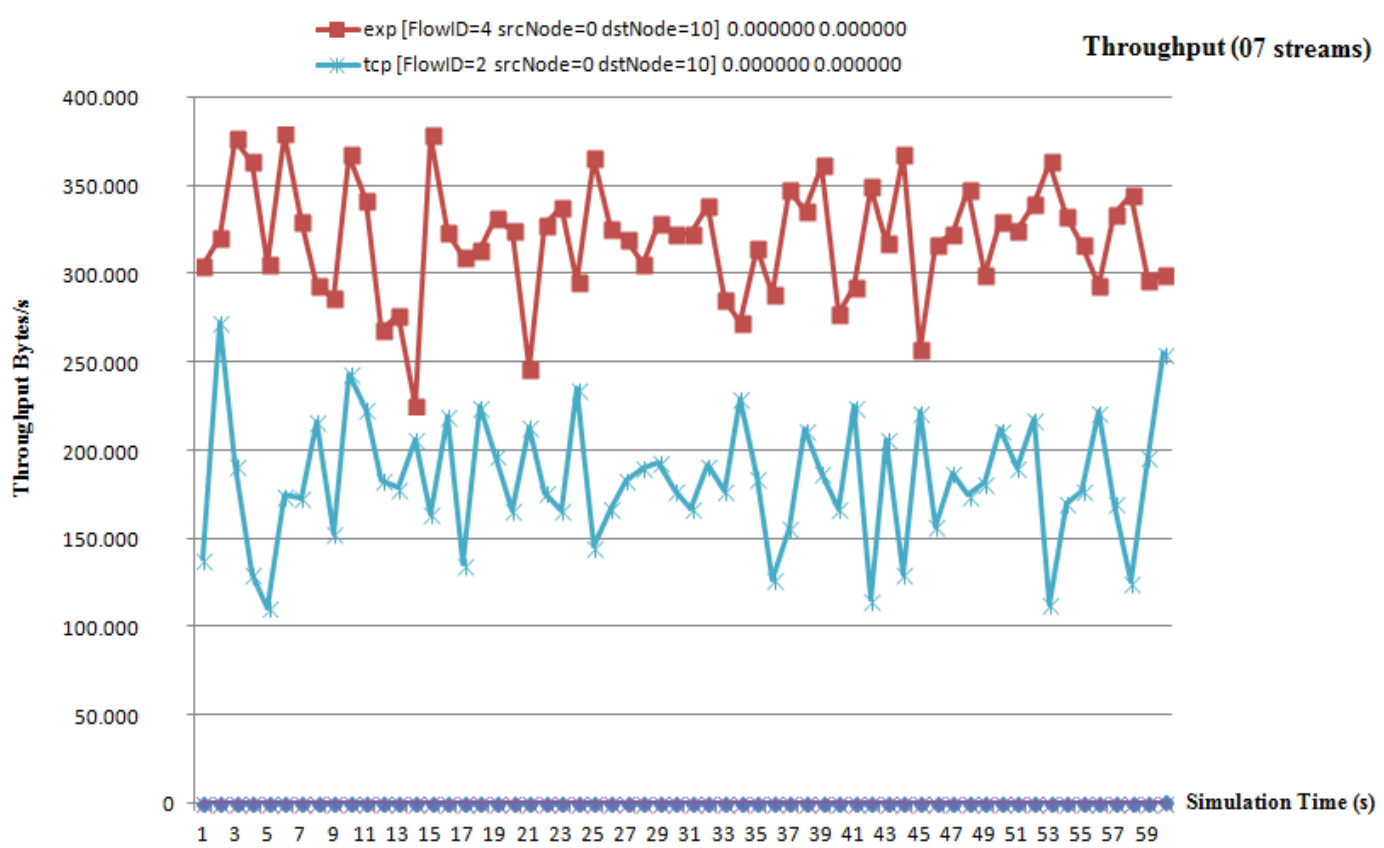

Fig. 7. Throughput IPTV x TCP (7 streams)

\section{Conclusion}

The work proposed had the finality to verify the viability or not of IPTV traffic insertion in the corporate network which could meet live television broadcast, in a satisfactory way and without significant losses of other applications already existing in the logical data network.
After the data collected and analyses made from these metrics, it can be conclude that, obeying the company current setting, it is viable the insertion of this new technology and platform. However, it is important to note that there is a very apparent limitation in the scalability of these streams. If there is a need of increasing the number of IPTV applications, fatally an 
investment will be necessary in the local infrastructure and in the backbone of this network. Besides, other Quality of Service strategies will be required to cover the new packet transmission needs.

Therefore, the work of research and previous simulations has a very great importance before the operation of these services. Furthermore, it will be necessary a cost/benefit study de implementation before the third-party company that provides the logical network backbone through a specific SLA.

Finally, television broadcast through data network has become a reality in the vast majority of large companies to support several business areas. It means that there will be real possibilities to bring even closer the managers and the operational areas, once possibilities of innovative technologies will be open as, for example, the IPTV applications in smartphones (mobile telephone with advanced functionalities). Consequently, the implementation viability of this platform in the company not only opens a wide range of possibilities of new services but also will put the company in a prominent level of innovation and digital inclusion.

\section{Author's Contributions}

Filipe Hiluy Lima: Undertake the required experiments and analyse the obtained results.

Henrique Mariano Costa do Amaral: Design the research and prepare the workflow.

Denílson Moreira Santos: Organizes the writing and structure of the manuscript.

\section{Ethics}

This article is original and contains unpublished materials. The corresponding author confirms that all of the other authors have read and approved the manuscript and no ethical issues involved.

\section{References}

Barros, J.LS, 2011. Proposta de Método para Análise Técnica de Rede para Implantação de Serviços IPTV. Escola Politécnica de São Paulo.

Reis Correa, B. and M. Sodré dos Reis, 2012. "IPTV: Protocolos Utilizados, Congresso de iniciação científica do INATEL-INCITEL, pp: 178-182.

Shihab, E. and L. Cai, 2007. IPTV distribution technologies in broadband home networks. Canadian Conference on Electrical and Computer Engineering, Apr. 22-26, IEEE Xplore Press, Vancouver, pp: 765-768. DOI: 10.1109/CCECE.2007.196

Tanenbaum, A.S., 2003. Redes de Computadores. 1st Edn., Campus-RJ, ISBN-10: 8535211853, pp: 945.

Uzunalioglu, H., 2009. Channel change delay in IPTV systems. Proceedings of the 6th IEEE Consumer Communications and Networking Conference, Jan. 10-13, IEEE Xplore Press, Las Vegas, pp: 1-6. DOI: $10.1109 /$ CCNC.2009.4784832

Vieira, O. and G. Maurício, 2010. Ferramenta para mapear a qualidade percebida pelo usuário em requisitos de QoS em ambiente convergente e heterogêneo. USP .

Wang, Y.S., K.H. Liang, H.C. Young and S.Y. Cheng, 2010. Quality-assured provisioning of IPTV services in ethernet-based broadband networks. Proceedings of the International Conference on Network and Service Management, Oct. 25-29, IEEE Xplore Press, Niagara Falls, pp: 266-269. DOI: 10.1109/CNSM.2010.5691197

Yarali, A. and A. Cherry, 2005. Internet Protocol Television (IPTV). Proceedings of the 10 Conference TENCON 2005 IEEE International Region, Nov. 21-24, IEEE Xplore Press, Melbourne, pp: 1-6. DOI: 10.1109/TENCON.2005.300861

Zeadally, S., H. Moustafa and F. Siddiqui, 2011. Internet Protocol Television (IPTV): Architecture, trends and challenges. IEEE Syst. J., 5: 518-526. DOI: 10.1109/JSYST.2011.2165601 